\title{
Humoral signatures of protective and pathological SARS-CoV-2 infection in children
}

\author{
Yannic C. Bartsch $\oplus^{1,10}$, Chuangqi Wang ${ }^{2,10}$, Tomer Zohar ${ }^{1,2}$, Stephanie Fischinger ${ }^{(1}{ }^{1}$, Caroline Atyeo ${ }^{1}$, \\ John S. Burke $\odot^{1}$, Jaewon Kang ${ }^{1}$ ', Andrea G. Edlow33, Alessio Fasano ${ }^{4,5}$, Lindsey R. Baden 6 , \\ Eric J. Nilles ${ }^{6}{ }^{6}$, Ann E. Woolley ${ }^{6}$, Elizabeth W. Karlson ${ }^{6}$, Alex R. Hopke ${ }^{7}{ }^{7}$, Daniel Irimia ${ }^{7}$, \\ Eric S. Fischer $\oplus^{8,9}$, Edward T. Ryan 7 , Richelle C. Charles $\oplus^{7}$, Boris D. Julg1, Douglas A. Lauffenburger $\left(^{2}\right.$, \\ Lael M. Yonker ${ }^{4,5} \bowtie$ and Galit Alter ${ }^{1 凶}$
}

\begin{abstract}
The severe acute respiratory syndrome coronavirus 2 (SARS-CoV-2) pandemic continues to spread relentlessly, associated with a high frequency of respiratory failure and mortality. Children experience largely asymptomatic disease, with rare reports of multisystem inflammatory syndrome in children (MIS-C). Identifying immune mechanisms that result in these disparate clinical phenotypes in children could provide critical insights into coronavirus disease 2019 (COVID-19) pathogenesis. Using systems serology, in this study we observed in 25 children with acute mild COVID-19 a functional phagocyte and complement-activating IgG response to SARS-CoV-2, similar to the acute responses generated in adults with mild disease. Conversely, IgA and neutrophil responses were significantly expanded in adults with severe disease. Moreover, weeks after the resolution of SARS-CoV-2 infection, children who develop MIS-C maintained highly inflammatory monocyte-activating SARS-CoV-2 IgG antibodies, distinguishable from acute disease in children but with antibody levels similar to those in convalescent adults. Collectively, these data provide unique insights into the potential mechanisms of IgG and IgA that might underlie differential disease severity as well as unexpected complications in children infected with SARS-CoV-2.
\end{abstract}

T he SARS-CoV-2 pandemic remains an enormous global challenge due to its persistent spread and unpredictable disease course. Although adults can exhibit moderate to severe disease with high mortality, children, surprisingly, experience largely benign disease characterized by mild cold-like symptoms despite high viral loads ${ }^{1-3}$. Several hypotheses for this disparate phenotype have emerged, including the presence of distinct angiotensin-converting enzyme 2 receptor expression patterns in children ${ }^{3}$, the potential for pre-existing cross-coronavirus (CoV) humoral immunity that might attenuate early infection ${ }^{4,5}$ and the potential for a more naive immune repertoire compared to adults. However, a small but significant fraction of children infected with or exposed to SARS-CoV-2 develop a Kawasaki disease (KD)-like syndrome, now termed MIS- ${ }^{6-8}$. Weeks after SARS-CoV-2 exposure or infection, children with MIS-C present with fever, clinical markers of inflammation and multi-organ involvement, which can include hypotensive shock, cardiac aneurysms and ventricular failure ${ }^{9}$

Emerging data have linked the humoral immune response to SARS-CoV-2 in adults to both protection and pathology ${ }^{10}$. To begin to define whether differences in the humoral immune response might account for differences in severity of disease across adults and children and point to pathological changes in MIS-C, in this study we deeply profiled the functional humoral immune response in 60 adults with acute SARS-CoV-2 infection (26 severe and 34 mild), 25 children with acute but mild SARS-CoV-2 infection and 17 children with polymerase chain reaction (PCR)- or serology-confirmed $(n=14)$ or suspected (for example, household contacts, $n=3$ ) SARS-CoV-2 exposure who developed MIS-C (11 severe and six mild). The data point to a pathological role for SARS-CoV-2-specific IgA responses linked to neutrophil activation in severe adult infection, which are not amplified in children or in mildly infected adults. Conversely, sustained levels of inflammatory macrophage-activating, Fc receptor-binding antibodies specific for different pathogens-including SARS-CoV-2-were selectively maintained in severe MIS-C disease. Collectively, these data identify unique functional antibody responses that implicate neutrophils and/or macrophages in SARS-CoV-2 disease pathology, revealing biomarkers and pathways to guide clinical care and therapeutic development.

\section{Results}

Within days of symptom onset, SARS-CoV-2-specific antibodies are detectable, marking SARS-CoV-2 exposure. However, beyond their absolute quantities, shifts in polyclonal antibody composition mark different responses to infection and disease trajectory ${ }^{11}$. Whether the polyclonal antibody composition differs across adults and children, the latter of whom experience largely asymptomatic

\footnotetext{
'Ragon Institute of MGH, MIT and Harvard, Cambridge, MA, USA. ${ }^{2}$ Department of Biological Engineering, Massachusetts Institute of Technology, Cambridge, MA, USA. ${ }^{3}$ Massachusetts General Hospital Department of Obstetrics and Gynecology, Division of Maternal-Fetal Medicine, Boston, MA, USA. ${ }^{4}$ Massachusetts General Hospital, Mucosal Immunology and Biology Research Center, Boston, MA, USA. ${ }^{5}$ Massachusetts General Hospital, Department of Pediatrics, Boston, MA, USA. ${ }^{6}$ Brigham and Women's Hospital, Boston, MA, USA. ${ }^{7}$ Massachusetts General Hospital, BioMEMS Resource Center, Boston, MA, USA. ${ }^{8}$ Department of Cancer Biology, Dana Farber Cancer Institute, Boston, MA, USA. ${ }^{9}$ Department of Biological Chemistry and Molecular Pharmacology, Harvard Medical School, Boston, MA, USA. ${ }^{10}$ These authors contributed equally: Yannic C. Bartsch, Chuangqi Wang. 凶e-mail: lyonker@mgh.harvard.edu; galter@mgh.harvard.edu
} 


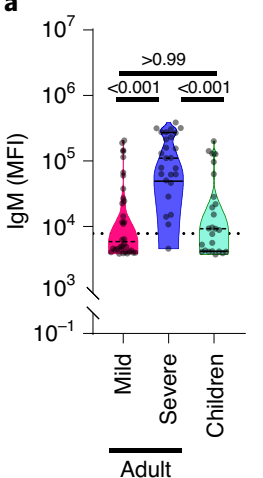

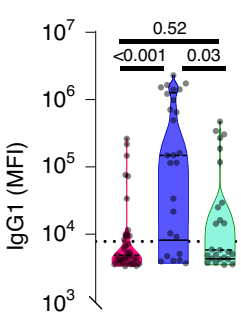

$10^{-1}$

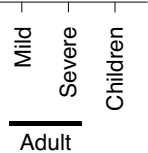

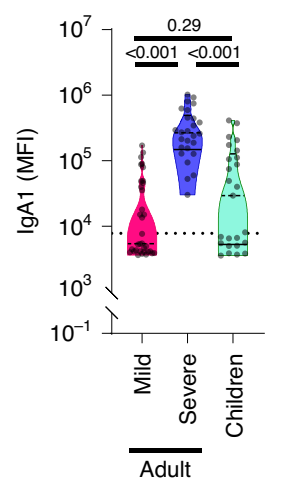

Adult_COVID severe
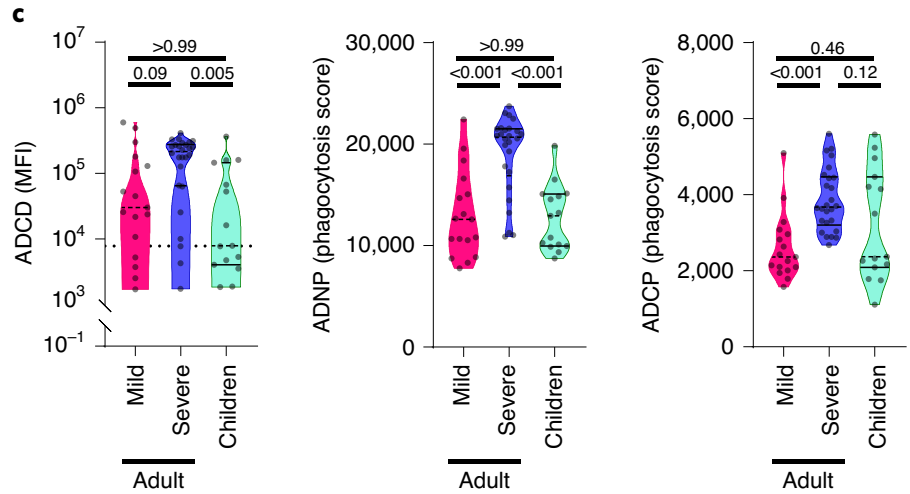

b

Children COVVID mild
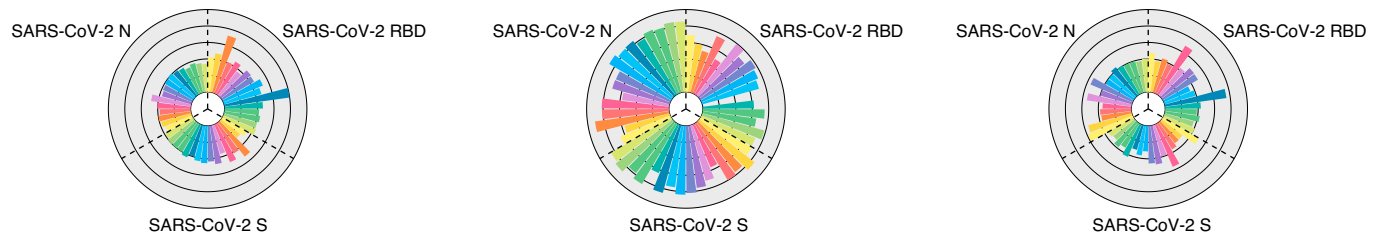

$\mathrm{Fc} \gamma \mathrm{R} 2 \mathrm{a}$ Fc $\gamma$ R2b $\mathrm{Fc} \gamma \mathrm{R} 3 \mathrm{a}$ Fc $\gamma$ R3b FC $\alpha R$ FcRn

Fig. 1 | Disease severity tracks with enhanced humoral immunity to COVID-19. SARS-CoV-2-specific plasma antibody responses in adult patients with mild (non-hospitalized) or severe (hospitalized) COVID-19 and pediatric patients with mild COVID-19 were analyzed. a, SARS-CoV-2 S protein-specific IgM, IgG1 and IgA1 titers were analyzed by Luminex $\left(n_{\text {mild }}=34, n_{\text {severe }}=26, n_{\text {children }}=25\right)$. The dotted line represents the average plus five times the s.d. of the negative plasma samples used to determine seropositivity (Methods). b, IgG subclasses 1-4, isotypes IgM, IgA1 and IgA2 and antibody-mediated functions (complement deposition (ADCD), neutrophil phagocytosis (ADNP) and THP-1 monocyte phagocytosis (ADCP)) for SARS-CoV-2 RBD, S and $\mathrm{N}$ were analyzed in all seropositive individuals $\left(n_{\text {mild }}=17, n_{\text {severe }}=26, n_{\text {children }}=15\right.$ ). Each flower plot summarizes the data from the respective group, and the length of each petal represents the average of the Z-scored value for the indicated feature (Supplementary Figs. 1-4). c, Univariate comparison of ADCD, ADNP and ADCP against SARS-CoV-2 S in seropositive individuals are shown. The dashed line in the violin plots indicates the median per group, and solid lines indicate quartiles. A non-parametric Kruskal-Wallis test was used to test for statistically significant differences between multiple groups. ADCP, antibody-dependent cellular phagocytosis. MFI, median fluorescence intensity.

to mild disease, is currently unknown. Thus, we comprehensively profiled the SARS-CoV-2-specific humoral immune response across a group of acutely infected adults and children, including 25 paucisymptomatic children and young adults (age 0-21 years, median $=15$ years) compared to 34 adults (age 22-76 years, median $=34$ years) with mild disease who did not require hospitalization and 26 adults (age 32-79 years, median $=56$ years) with more severe SARS-CoV-2 infection, all of whom required hospitalization (Supplementary Table 1). Acute SARS-CoV-2 infection was diagnosed by related symptoms and positive PCR and/or serology. Samples were collected 0 $-52 \mathrm{~d}$ after symptom onset (median $=9 \mathrm{~d}$ ), depending on patient availability (for example, during outpatient clinic visit).

Low IgA and phagocytic activity track with mild disease in children and adults. As expected, high titers of SARS-CoV-2 spike (S)-specific IgM, IgG and IgA titers were observed in adults with severe infection (Fig. 1a,b). Conversely, S-specific IgG and IgM levels were similar in paucisymptomatic children and adults with mild acute infection but significantly lower compared to severe disease. In contrast to more attenuated but similar SARS-CoV-2 receptor-binding domain (RBD), S- and nucleocapsid (N)-specific isotype, subclass and $\mathrm{Fc}$ receptor-binding levels in children and adults with mild disease, severely ill patients exhibited robustly expanded humoral immune responses (Fig. 1b and Supplementary Figs. 1-3). Next, we tested the ability of plasma antibodies to induce Fc-mediated effector function. Antigen:antibody immune complexes (ICs) were generated by incubating plasma with antigencoated fluorescent beads, and ICs were then cultured with complement, THP-1 monocytes or HL60-differentiated neutrophils to analyze complement deposition or phagocytic activity. Similar levels of complement, neutrophil and monocyte-activating antibodies were observed in children and mildly infected adults but were significantly lower than in adults with severe infection (Fig. 1c and Supplementary Fig. 4). Interestingly, pediatric patients, typically diagnosed earlier, exhibited a tendency to develop antibodies earlier after symptom onset compared to adults. Within $7 \mathrm{~d}$ after symptom onset, only two of 18 adults versus five of 18 children were positive for S-specific IgG1, and only three of 18 adults versus seven of 18 children were positive for S-specific IgM, potentially contributing to reduced pathology and differences in kinetics of overall symptomatology across the ages (Supplementary Fig. 5); however, responses in children were similar to those observed in adults who experienced mild disease (Fig. 1a,b). These data suggest that children might not necessarily make a stronger or more effective functional humoral immune response compared to adults.

To next determine whether any multivariate differences exist among the adult and pediatric profiles of seropositive individuals, an unsupervised uniform manifold approximation and projection (UMAP) was initially used to collapse the multivariate data into two dimensions, with proximity representing similarities in the overall dataset. Separation was observed visually across children and severely ill individuals in the UMAP, pointing to substantial global humoral profile differences across the groups (Fig. 2a). Similarly, discrete antibody profiles were observed across severely and mildly ill adults (Fig. 2b). In contrast, adults with mild disease and children overlapped extensively, highlighting the multivariate similarity in the overall SARS-CoV-2 antibody profiles between the groups (Fig. 2c). The integration of all three groups in a single UMAP 
a

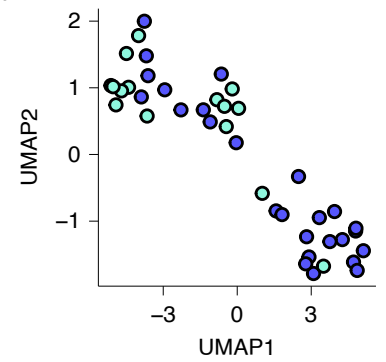

b

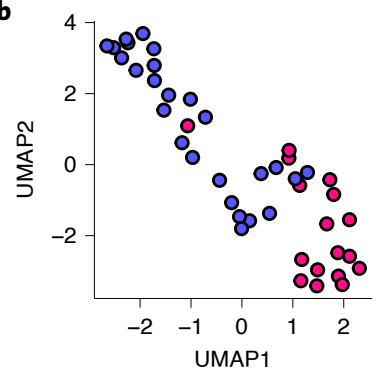

c

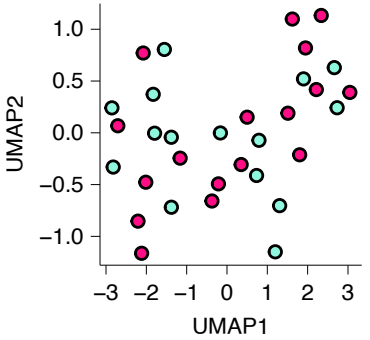

d

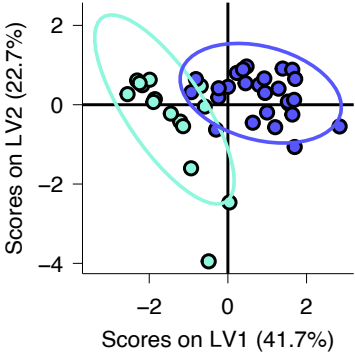

e

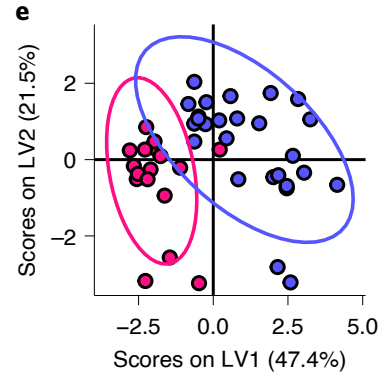

$\mathbf{f}$

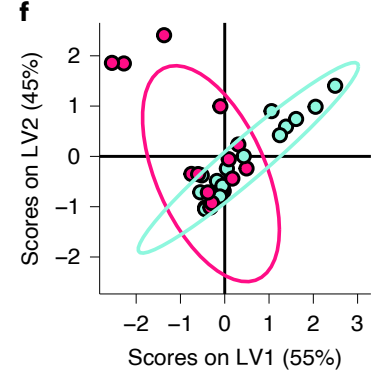

g
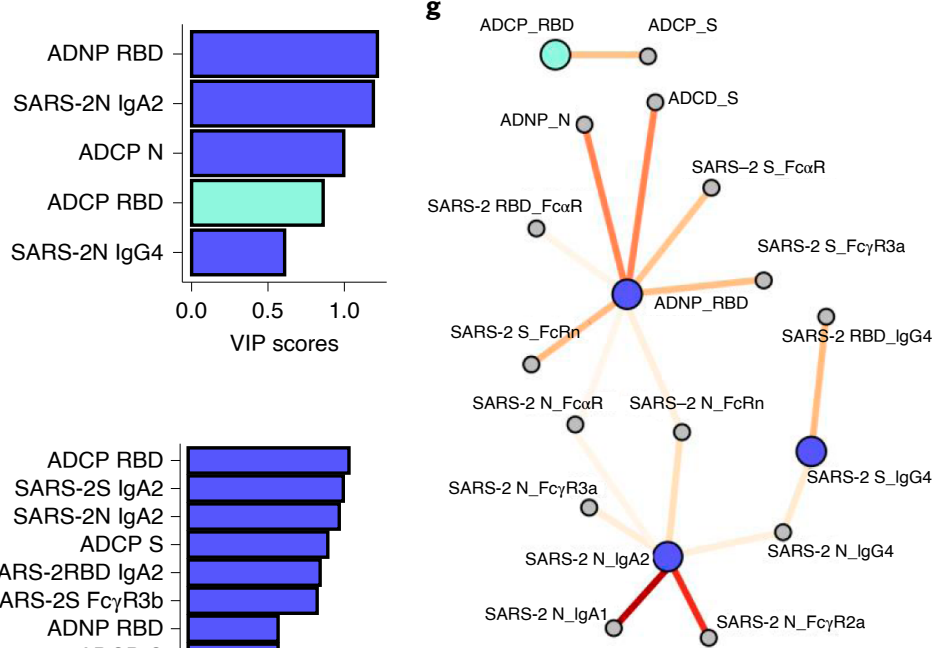

Fig. 2 | SARS-CoV-2-specific IgA titers and augmented antibody functionality discriminate severe from mild disease. Pairwise comparisons were performed between seropositive children with mild disease and severely ill adults ( $\mathbf{a}$ and $\mathbf{d}$ ), adults with mild and severe disease (b and e) and children and adults with mild disease ( $\mathbf{c}$ and $\mathbf{f})\left(n_{\text {mild }}=17, n_{\text {severe }}=26, n_{\text {children }}=15\right)$. $\mathbf{a}-\mathbf{c}$, Multivariate UMAP analyses show the variation in the humoral profiles across the groups. Proximity of points indicate homology in the overall dataset. d-f, In a second approach, a supervised multivariate comparison was performed across groups, where features were initially reduced using LASSO, to avoid overfitting, and then visualized by PLS-DA (left panel). Cross-validation accuracy for d-f was 0.91, 0.97 and 0.65, respectively. The LASSO-selected features were also plotted and ranked in a VIP score plot (right panel; color of bars indicate in which group the feature was enriched). $\mathbf{g}$, Network correlations depict the additional non-LASSO-selected Fc profile features (small circles) that were correlated with the LASSO-selected features (big circles) based on pairwise comparisons between children and severely ill adults. The connections between points (features) indicate significant $(P<0.05)$ Spearman correlations. The fill color of the circles indicates in which group the selected feature were enriched (gray indicates that the feature was not selected); the color of the connecting lines indicates the strength of the correlation coefficient. LV, latent variable; VIP, variable importance in projection.

further highlighted the similarity in mild disease antibody profiles among adults and children that were largely distinct from, albeit along a continuum with, those in severely ill adults, suggesting that severity of disease, rather than age, might influence antibody profiles predominantly (Supplementary Fig. 6).

To further define the individual features that distinguished children or adults with mild infection from adults with severe disease, we used least absolute shrinkage and selection operator (LASSO) feature selection to initially reduce the total 48 antibody variables to a minimal set that represented the overall variation in the antibody profiles, followed by classification using partial least squares discriminant analysis (PLS-DA). Nearly perfect separation was observed between children and severely ill adults (Fig. 2d). The model used only five of the total 48 measured features to resolve the two groups, including RBD-specific neutrophil phagocytosis, N-specific IgA2 levels and monocyte phagocytosis enriched in severely ill adults and RBD-specific monocyte phagocytosis enriched in children. These data point to significantly expanded IgA and phagocytic functional responses in severely ill individuals, suggesting IgA-driven IC phagocyte activation as a marker of disease severity. Likewise, near complete separation was observed between adults with mild and severe disease (Fig. 2e), marked, again, by high levels of monocyte phagocytic functions, IgA2 responses and $\mathrm{S}$-specific binding to Fc $\gamma \mathrm{R} 3 \mathrm{~b}$ expressed on neutrophils, neutrophil phagocytosis and complement activation and IgM titer in severely ill adults. In contrast, antibody profiles between children and adults with mild disease could not be resolved (Fig. 2f).

A correlation network was constructed between LASSO-selected features and the overall immune response to deeply probe the distinct relationships that diverged between severe and mild disease (Fig. 2g and Supplementary Fig. 7). N-specific IgA, N-, S- and RBD-specific $F c \alpha$ receptor (Fc $\alpha \mathrm{R}$ ) binding (found constitutively on neutrophils ${ }^{12}$ ), neutrophil phagocytosis and complement activation were all induced in a synchronized manner, pointing to a central axis of IgA:neutrophil activation in severe disease. Moreover, whereas depletion of IgG, our dominant serum antibody, resulted in 
attenuated neutrophil phagocytosis across all groups, depletion of IgA reduced antibody-dependent neutrophil phagocytosis (ADNP) significantly only among severely ill individuals (Fig. 3a-c). In addition, whereas depletion of IgG was sufficient to eliminate ADNP in adults and children with mild disease (Fig. 3a,c), combined depletion of IgG and IgA resulted in complete loss of ADNP in adults with severe disease (Fig. 3c) and pointed to an added role of IgA in neutrophil activation in severe disease.

Given our emerging appreciation for excessive neutrophil activity in COVID-19 pathology ${ }^{13,14}$, we further probed the functional role of IgA in neutrophil IC responsiveness in vitro. Despite the pronounced effect of IgG in attenuating neutrophil phagocytosis, the depletion of both $\operatorname{IgG}$ and $\operatorname{IgA}$ resulted in a loss of degranulation-mediated release of myeloperoxidase (MPO), lactoferrin and matrix metalloproteinase-9 (MMP-9), as well as attenuated cytokine secretion (Fig. 3d and Supplementary Fig. 8). Specifically, compared to IgG depletion, elimination of IgA further decreased tertiary MMP-9 granule, interleukin (IL)-1 $\beta$, IL-6 and IL-8 release from neutrophils (Fig. $3 \mathrm{~d}$ ), resulting in a unique IgA-driven multivariate functional cascade marked by enhanced inflammatory neutrophil activity in the presence of SARS-CoV-2-specific IgA (Fig. 3e), as has been noted previously ${ }^{15,16}$. Furthermore, longitudinal profiling of an orthogonal cohort of hospitalized individuals pointed to the selective evolution of enhanced IgA2 levels in severely ill individuals, particularly 1 week after symptom onset, linked to disproportionately higher levels of FcoR binding and ADNP activity (Fig. 3f), potentially implicating IgA2 emergence as a unique mechanistic marker of severe SARS-CoV-2 disease. Thus, collectively, these data strongly implicate IgA-driven functions in severe disease that do not evolve in children or adults with mild disease. However, although exploratory, additional investigation is needed to completely resolve the contribution of IgA-driven neutrophil activation to the full severity cascade observed in severe SARS-CoV-2 infection.

Persistence of inflamed SARS-CoV-2 antibodies track with MIS-C. Despite the largely mild nature of pediatric SARS-CoV-2 infection, a subset of children develop a severe inflammatory-mediated illness after infection, MIS-C, including potential antibody-driven immunopathology similar to $\mathrm{KD}^{6,8}$ Although early data has pointed to differences in IgM levels in children with MIS-C compared to acutely infected adults and children ${ }^{17}$, this loss of IgM could simply be attributable to the resolution of infection in MIS-C that occurs weeks after infection. Thus, to begin to delineate whether particular polyclonal antibody signatures could provide mechanistic insights into MIS-C, SARS-CoV2 -specific humoral immunity was profiled across children with mild $(n=6)$ or severe $(n=11)$ MIS-C compared to a group of non-hospitalized convalescent adults $(n=18)$ who had mild disease symptoms (not hospitalized and no oxygen therapy required), the latter matched for time from suspected infection (days from COVID-19 symptom onset: 11-38, median: 27 d) (Fig. 4 and Supplementary Table 1). A total of 17 children and young adults (0-21.9 years, median: 3.5 years, $83 \%$ male, days from MIS-C symptom onset: $2-23$, median: $3 \mathrm{~d}$ ) were diagnosed with MIS-C per Centers for Disease Control and Prevention (CDC) criteria (https:// www.cdc.gov/mis-c/hcp/): patients had fever, laboratory evidence of inflammation or multi-system involvement that required hospitalization, with no alternative diagnosis. In addition, all patients with MIS-C had evidence of SARS-CoV-2 infection (positive SARS-CoV-2 by RT-PCR or antigen test or positive SARS-CoV-2 antibodies) or confirmed SARS-CoV-2 exposure (for example, household or childcare contacts). Of note, one of our patients was 21.9 years old but otherwise met CDC MIS-C criteria. Patients with MIS-C were stratified by severity, with patients with severe disease defined as experiencing cardiac complications (Methods and
Supplementary Table 2). The severe MIS-C cohort exhibited similar IgM and IgG1 titers to those observed in convalescent adults. Conversely, despite a positive RT-PCR result in $50 \%$ of the children, SARS-CoV-2 antibodies were not detectable in most patients with mild MIS-C here (only one child in this group had detectable levels of S- and RBD-specific IgG3 and IgA1 by Luminex; Fig. 4a and Supplementary Figs. 1-3). However, all patients with MIS-C had evidence of previous SARS-CoV-2 exposure defined by either positive PCR or serology or confirmed contact. Focusing on convalescent adults and children with severe MIS-C who both harbored robust SARS-CoV-2 humoral immune responses weeks after infection, a broad isotype, subclass and functional antibody profile was observed (Fig. 4b and Supplementary Fig. 4), although the specific expanded humoral features differed across the groups (Fig. 4b). For example, although similar levels of complement deposition and neutrophil phagocytosis were observed in the convalescent adults and patients with severe MIS-C (Fig. 4b,c), patients with MIS-C exhibited enhanced monocyte-activating capacity (Fig. 4c). This suggests convalescent-like profiles in the patients with severe MIS-C but the presence of a select set of altered antibody features that uniquely emerge in this pathological setting.

To gain deeper insights into the specific immune alterations that define MIS-C, we next performed multivariate analysis. Clear separation was observed in the multivariate SARS-CoV-2 response in mild and severe MIS-C (Fig. 5a) by both UMAP and LASSO/PLS-DA, driven by the presence of SARS-CoV-2-specific IgG and IgM responses in the severe cases. Interestingly, patients with severe MIS-C were distinguishable from both convalescent adults (Fig. 5b) and children with mild disease (Fig. 5c), largely linked to higher SARS-CoV-2-specific antibody titers with more phagocytic and functional activity distinct from immune responses in severe acute disease in adults (Supplementary Fig. 9). Network analysis highlighted the over-representation of functional humoral immune responses in the children with MIS-C, pointing to a potential IC-based activity in disease pathology (Fig. $5 \mathrm{~d}$ ). Additionally, comparison of children with MIS-C to adults with severe acute disease revealed distinct antibody profiles across the two groups, with expanded IgG-driven function in MIS-C and $\mathrm{Ig} \mathrm{A} /$ neutrophil-expanded immune responses in the adults with severe disease, pointing to a persistent IgG-related disease in MIS-C (Supplementary Fig. 10) ${ }^{17}$ and IgA-related disease in acute SARS-CoV-2 infection.

However, why the children with MIS-C maintained a response able to continue to recruit a SARS-CoV-2-specific phagocytic and complement activating response remains unclear but collectively points to more inflamed antibody profiles among children with severe MIS-C. Strikingly, this inflamed antibody profile was not only observed in the SARS-CoV-2-specific humoral immune response: as shown in Fig. 5e (and Supplementary Fig. 11), an expansion of highly functional antibodies was observed specific for multiple pathogens that have been implicated in, but not proven to drive, KD (Epstein-Barr virus (EBV), measles, endogenous retrovirus (ERV), Bordetella pertussis and Staphylococcus aureus), auto-antigens linked to MIS-C, common respiratory pathogens (influenza and respiratory syncytial virus (RSV)) as well as common CoVs (strains: OC43, 229E, HKU1 and NL63) ${ }^{18,19}$. Although no differences were observed in overall IgG levels (Supplementary Fig. 12), these findings point to a significantly expanded Fc receptor-binding humoral antibody repertoire among children with severe MIS-C but not in adults with severe acute disease (Fig. 5e and Supplementary Fig. 11). In the absence of hypergammaglobulinemia, this points to a significant non-specific amplification of functional pathogen-specific IgG-driven immunity, able to drive broad inflammation in severe MIS-C. Together, these data argue for a generalized dysregulated pro-inflammatory IgG response to SARS-CoV-2 and beyond in severe MIS-C. 
a

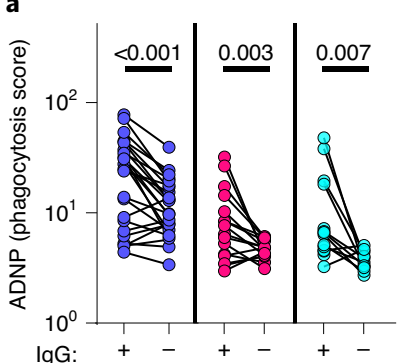

b

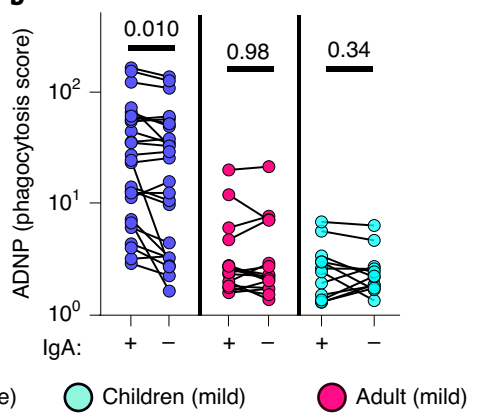

c

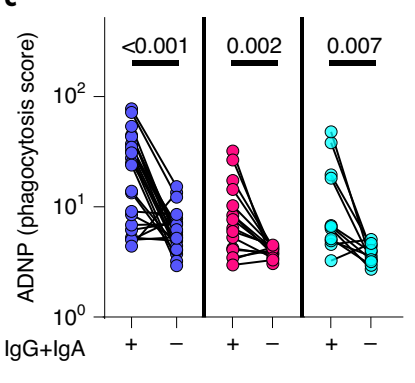

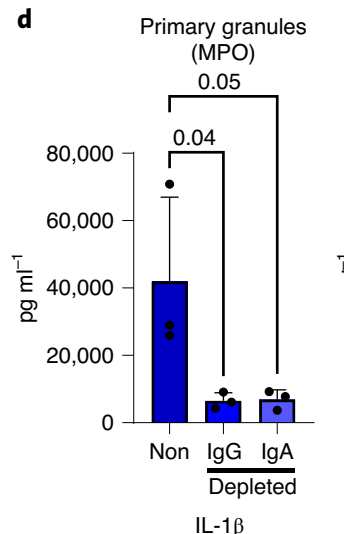

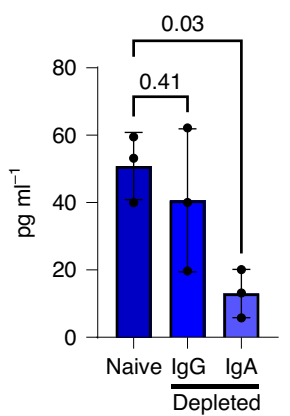

Adult (ser
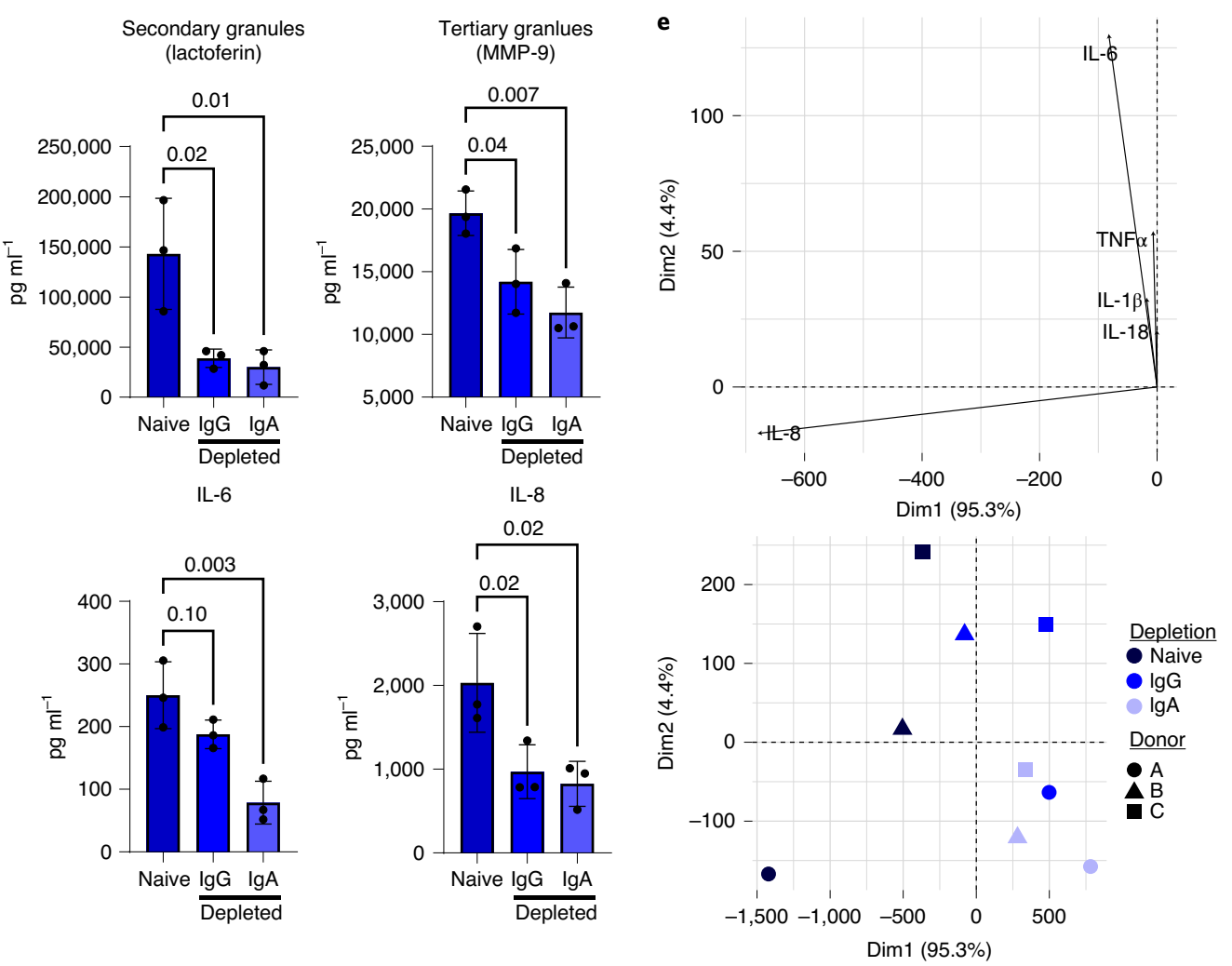

f $\lg \mathrm{A} 1$

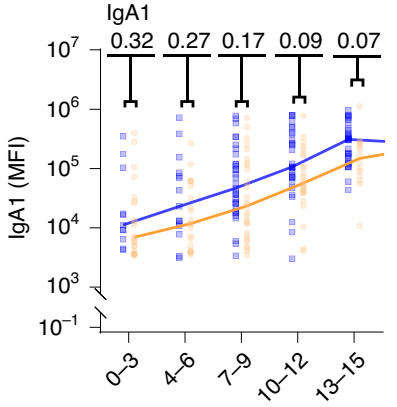

Days after symptom onset

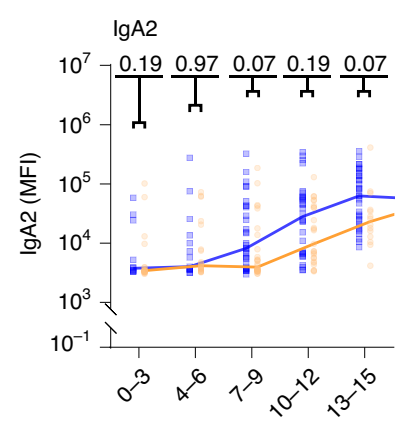

Days after symptom onset

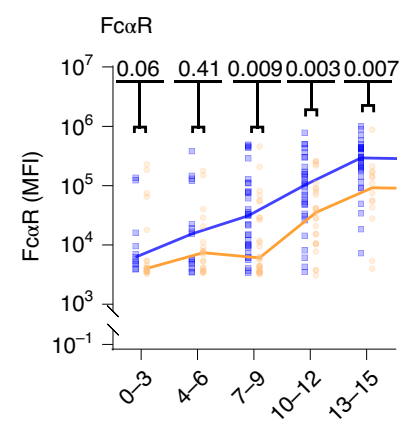

Days after symptom onset

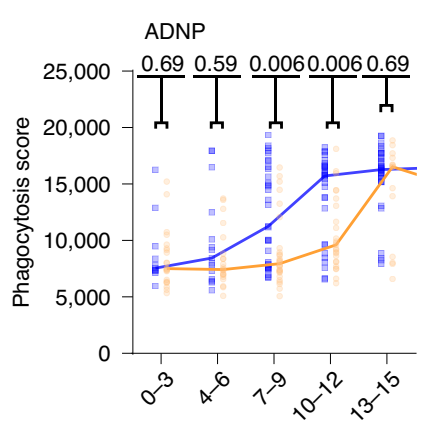

Days after symptom onset

Fig. 3 | Unique functional and evolutionary profiles of SARS-CoV-2 IgA antibodies. The matched-line graphs show the effect of $\lg G(\mathbf{a}), \operatorname{lgA}(\mathbf{b})$ and IgG+lgA (c) depletion on ADNP activity across severely ill adults (dark blue, $n=25$ ), mildly ill adults (red, $n=16$ ) and children with mild disease (light blue, $n=12)$. d, The bar graphs show the level of degranulation of MPO, lactoferrin and MMP-9 and cytokine secretion of IL-1 $\beta$, IL- 6 and IL-8 in pooled naive/non-depleted (Non) plasma from severely ill adult patients with COVID-19 compared to IgG- or IgA-depleted plasma. Data are presented as mean values \pm s.d. of three individual blood neutrophil donors. e, Principal component analysis highlights the multivariate functional profiles observed in non-depleted, IgG- or IgA-depleted plasma, highlighting the distinct inflammatory cascades associated with IgA depletion (light blue) that intermingled with IgG-depleted plasma (medium blue), both of which were distinct from naive/undepleted plasma (black). $\mathbf{f}$, The line graphs show the longitudinal evolution of IgA1, IgA2, FcaR binding and ADNP at different time points in severely ill $(n=59)$ or moderately ill $(n=77)$ individuals followed within the first 2 weeks after symptom onset (not all time points for all individuals were available). Statistical significance in a-c was calculated by non-parametric, two-sided Wilcoxon matched-pairs signed-rank test and a parametric one-way analysis of variance in $\mathbf{d}$. A Mann-Whitney test was used to assess statistical differences between the groups at each interval in $\mathbf{f}$, and $P$ values were corrected for multiple hypothesis testing using the Benjamini-Hochberg procedure. Dim, dimension (principal component); MFI, median fluorescence intensity; TNF, tumor necrosis factor. 

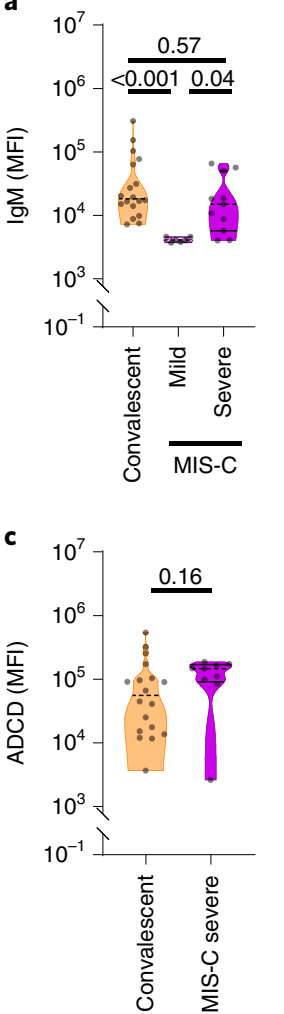

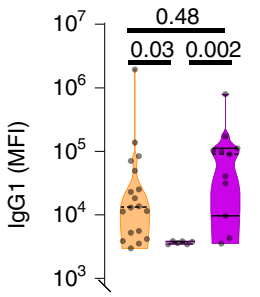

$10^{-1}$
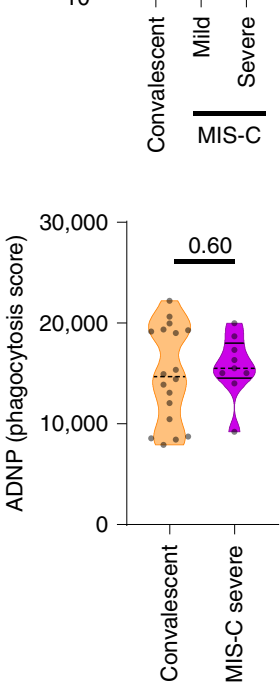

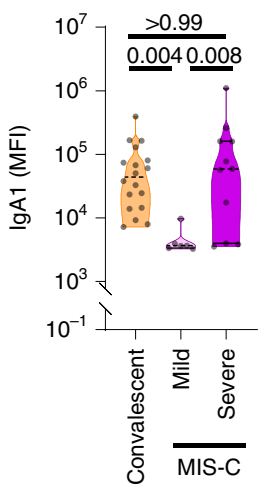

b

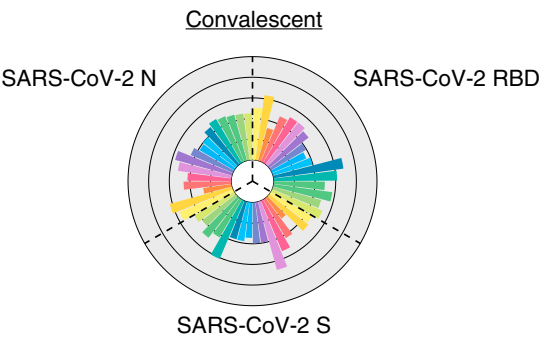

MII

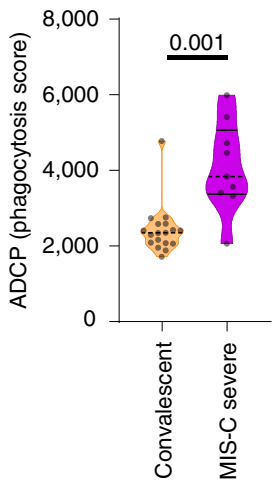

SARS-COV-2 RBD

Fig. 4 | Distinct SARS-CoV-2-specific antibody responses in children with severe MIS-C. SARS-CoV-2-specific plasma antibody responses were analyzed in children with mild or severe MIS-C or convalescent adults. a, SARS-CoV-2 S protein-specific IgM, IgG1 and IgA1 titers were analyzed by Luminex ( $n_{\text {convalescent }}=18, n_{\text {mild }}=6, n_{\text {severe }}=11$ ). The dotted line represents the average plus five times the s.d. of the negative plasma samples used to determine seropositivity (Methods). b, IgG subclasses 1-4, isotypes IgM, IgA1 and IgA2 and antibody-mediated functions (ADCD, ADNP and ADCP) for SARS-CoV-2 $\mathrm{RBD}, \mathrm{S}$ and $\mathrm{N}$ in all seropositive individuals were analyzed ( $n_{\text {conv }}=18, n_{\text {severe }}=9$ ). Each flower plot summarizes the data of the respective group, and each petal represents the average of the Z-scored value for the indicated feature (Supplementary Figs. 1-4). c, Univariate analysis of ADCD, ADNP and ADCP against SARS-CoV-2 S are shown. Dashed lines in the violin plots indicate the median per group, and solid lines indicate quartiles. A non-parametric, two-sided Mann-Whitney test was used to test for statistically significant differences between the two groups. ADCP, antibody-dependent cellular phagocytosis. MFI, median fluorescent intensity.

\section{Discussion}

The unpredictable nature of SARS-CoV-2 disease severity is alarming, with severe acute disease emerging in adults and a KD-like MIS-C in children ${ }^{8}$. In the absence of therapeutics able to reverse these clinical manifestations, understanding the immunological mechanisms that underlie these unusual complications of SARS-CoV-2 infection might provide critical insights for the design and delivery of therapeutics for these unique populations. Here we note divergent but unique humoral immune profiles among mild symptomatic children and young adults and children with severe MIS-C that implicate distinct humoral immune functions in disease pathology. The induction of SARS-CoV-2-specific IgA responses, linked to neutrophil activation, was enriched in severely ill adults but was less pronounced in the humoral immune response in children and mildly affected adults. Conversely, children who developed MIS-C exhibited persistent, enhanced Fc receptor-binding antibodies, capable to recruit monocytes in a selective manner.

Beyond the pivotal role of IgG-mediated functions, IgA is the most highly produced antibody isotype in the body $\left(66 \mathrm{mg} \mathrm{kg} \mathrm{kg}^{-1}\right.$ $\mathrm{d}^{-1}$ ), and, although highly enriched at mucosal surfaces ${ }^{20}$, serum IgA has emerged as a highly potent activator of innate immune effector function ${ }^{21}$. In the blood, ICs including IgA interact with Fc $\alpha$ R, constitutively expressed on neutrophils, but also upregulated on several mucosal immune cell subsets. Although neutrophils also express Fc receptors for IgG, both IgA and IgG result in different neutrophil functions, where IgG drives phagocytosis and cytokine secretion, and IgA can augment these functions and additionally drive robust degranulation, cytokine secretion and NETosis ${ }^{22}$. Along these lines, depletion of both IgG and IgA in severely affected adults resulted in attenuated neutrophil activation; however, the depletion of IgA resulted in more robust attenuation of tertiary degranulation and cytokine release. Thus, in addition to IgG, elevated $\operatorname{IgA}$ levels, observed in adults with more severe disease ${ }^{23}$, might be linked to enhanced disease severity via the recruitment of $F c \alpha R$-driven inflammatory cascades downstream of phagocytosis (Fig. 1 and Supplementary Figs. 1-3). Given the presence of functional IgG, but not IgA, responses in both children and adults with mild disease, the data potentially argue that IgG alone might be sufficient to control and contain the infection in mild infection. Our work, exploiting patient-derived antibodies and neutrophils derived from healthy individuals, might not recapitulate the full spectrum of the antibody/innate cascade. In fact, significant changes have been noted in the frequency ${ }^{24}$, distribution ${ }^{25}$ and function ${ }^{13,14}$ of neutrophils across the SARS-CoV-2 spectrum of disease. Thus, future studies focused on the autologous interaction of ICs and activated innate immune cells might reveal critical insights related to the fully pathological cascade that might tip the response from immunoprotective to immunopathological.

Although IgA could simply represent a biomarker of enhanced viremia in the lung, it is thought to play a critical role in 

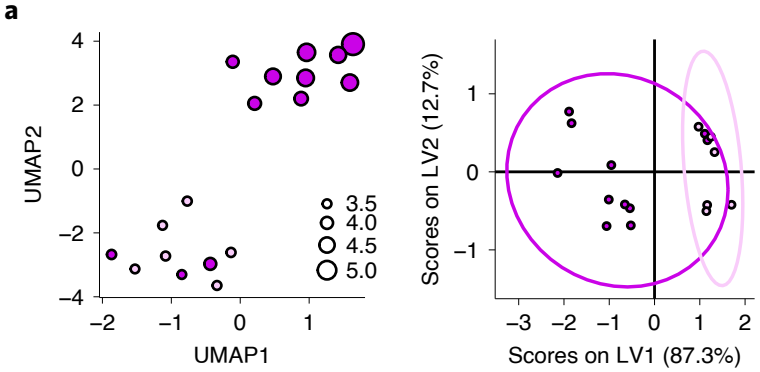

b
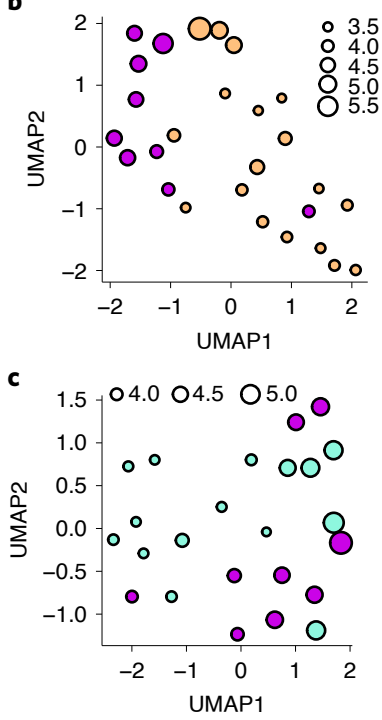

e

SARS2 ${ }^{-}$children
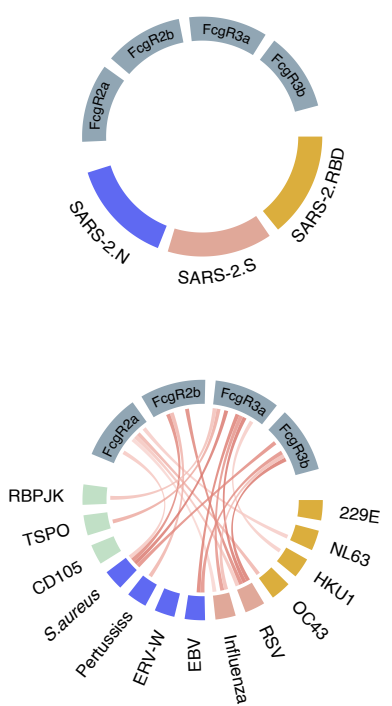
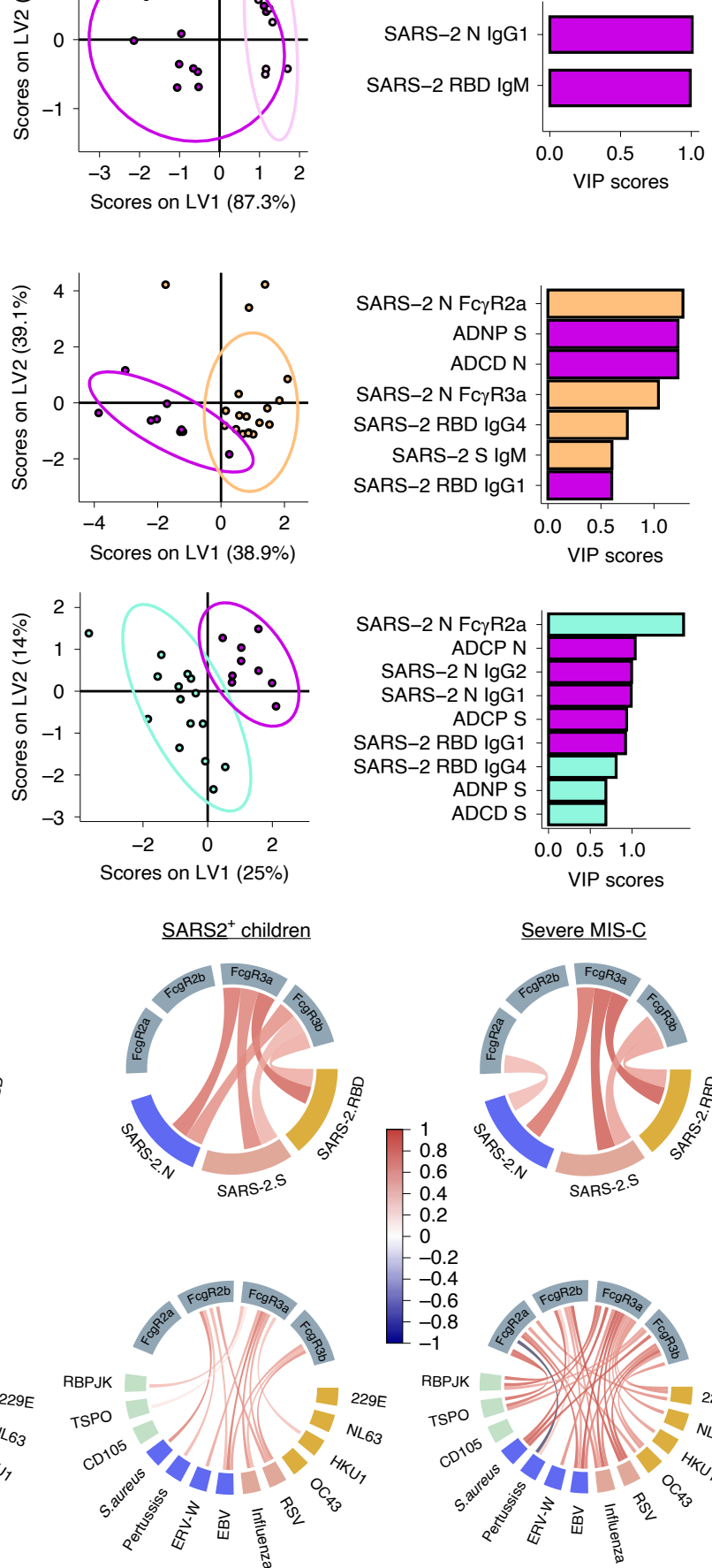

Severe MIS-C
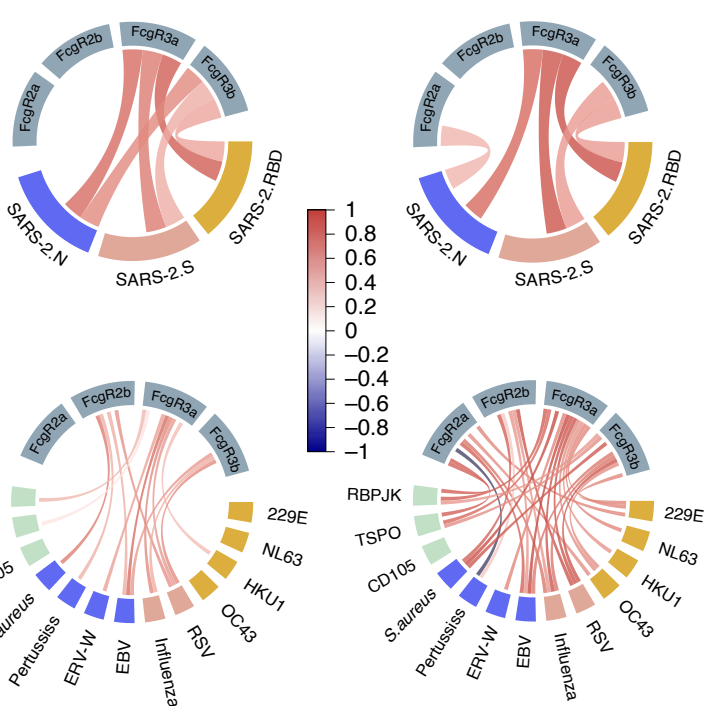
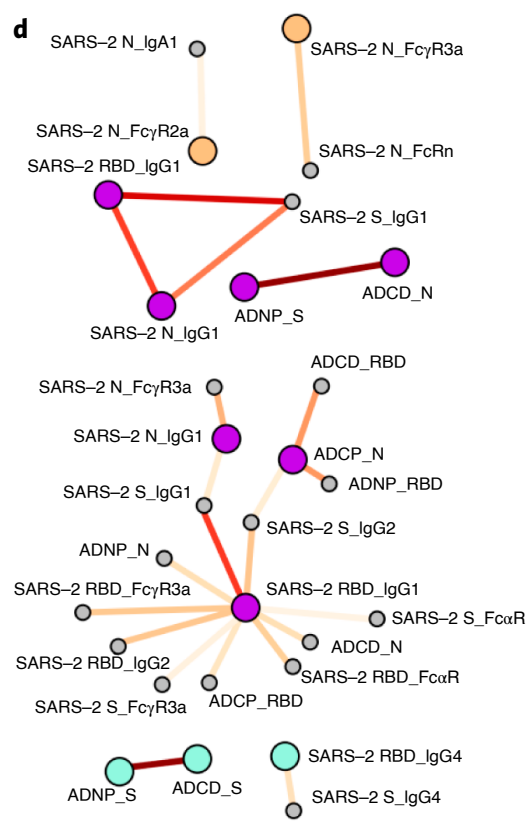

Spearman correlation $\quad$ Model selected

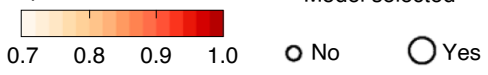

MIS-C severe

Mild MIS-C

O SARS2 ${ }^{+}$children

Convalescent

Fig. $\mathbf{5}$ | Dysregulated and pro-inflammatory antibody profiles in children with severe MIS-C. Pairwise comparisons by UMAP and LASSO/PLS-DA analyses are shown across mild and severe MIS-C (a), severe MIS-C and convalescent $(\mathbf{b})$ and severe MIS-C and acutely infected children $(\mathbf{c})\left(n_{\text {mild }}\right.$ MIs-c $=6$, $n_{\text {severe MIS-C }}=9, n_{\text {SARS } 2}{ }_{\text {children }}=15, n_{\text {convalescent }}=18$ ). The size of each dot represents the $\log _{10}$-scaled value of lgG1 titer to SARS-CoV-2 RBD. The cross-validation accuracy in $\mathbf{b}$ and $\mathbf{c}$ is 0.89 and 0.88 , respectively. $\mathbf{d}$, The correlation network of LASSO-selected (big circles) or unselected (small circles) features are shown across children and severely ill adults. Connection between points (features) indicate significant relationships $(P<0.05)$ defined by Spearman correlation after Benjamini-Hochberg correction. Fill color of the circles indicates in which group the selected feature was enriched (gray indicates that the feature was not selected), and the color of the connecting lines indicates the correlation coefficient. $\mathbf{e}$, The chord diagrams show the relationships of Luminex-defined IgG1 titers across SARS-CoV-2 antigens (upper panel: yellow, RBD; red, S; blue, N) and other pathogens and auto-antigens (lower panel: yellow, common CoV RBDs; red, other respiratory viruses; blue, KD-associated pathogens; green, MIS-C-associated auto-antigens) assessed by Spearman correlation. The connecting lines between colored boxes (antigen-specific lgG1 titer) and gray boxes (FcyRs) indicate a significant $(P<0.05)$ correlation between antigen-specific lgG1 titers and binding to the indicated Fc $\gamma R$ for the respective antigen. A color gradient was used to indicate the correlation coefficient (from $r=-1$ (dark blue) over $r=0$ (white) to $r=1$ (dark red)) for the individual correlations. LV, latent variable; VIP, variable importance in projection. 
mucosal immunity. However, the potent inflammatory activity of IgA might also contribute to enhanced inflammation and symptomatic disease in adults. Interestingly, sero-surveillance studies in otherwise healthy children have shown that IgA levels increase with age, peaking in the late teenage years in the blood ${ }^{26}$. Conversely, children have been noted to have more severe disease after other respiratory infections, including influenza, pertussis, RSV and group B streptococci ${ }^{27}$, hypothesized to be related to the lower capacity to generate IgA. Although it is critical to note that children with MIS-C generated SARS-CoV-2 IgA responses, these responses tended to be lower than those observed in adults (Supplementary Figs. 1-4). Thus, collectively, these data might point to a potentially unexpected pathological role of IgA in adult SARS-CoV-2 infection that might contribute to enhanced disease in the elderly via the over-activation of neutrophil degranulation and inflammation.

Children diagnosed with MIS-C, conversely, exhibited a persistence of functional SARS-CoV-2-specific monocyte-activating antibodies and expanded pan-pathogen FcyR-binding antibodies, in the absence of hypergammaglobulinemia (Supplementary Fig. 12). Given that monocyte activation and ICs have both been associated with KD disease severity ${ }^{28,29}$, these data point to potential mechanistic disease-associated parallels between KD and MIS-C. Moreover, similarly to enhanced Fc $\gamma$ R2a binding observed here in MIS-C, $\mathrm{F} c \gamma \mathrm{R} 2 \mathrm{a}$, a receptor essential for monocyte phagocytosis ${ }^{30}$, has been described as a susceptibility locus for $\mathrm{KD}^{31}$. Furthermore, effective intravenous immunoglobulin (IVIG) treatment of KD has been linked to direct inhibition of Fc $\gamma$ R2a-mediated IC activation $^{32,33}$, further supporting the importance of FcyR-binding antibodies in this pediatric disease.

As reported for other MIS-C cases $^{34}$, none of our patients with mild MIS-C had detectable SARS-CoV-2 titers in our hands but experienced more symptoms, including cough, compared to patients with severe MIS-C (three of six versus two of 11 in severe MIS-C). Conversely, as one would expect from a previous exposure, most of our patients with severe MIS-C were seropositive for SARS-CoV-2. Two children lacked SARS-CoV-2-specific antibodies; thus, we cannot exclude that these were KD cases. However, most notable was the persistence of SARS-CoV-2 immunity among the patients with severe MIS-C over time (Supplementary Fig. 10) ${ }^{17}$, in contrast to emerging reports of rapid humoral waning in both symptomatic and asymptomatic adults ${ }^{35,36}$. Whether persisting functional antibodies are the result of sustained viral replication in these children; are related to an aberrant ongoing germinal center reaction; are related to durable specific ICs; or are related to a general activation of the humoral immune response remains unclear. However, a recent report of non-specific plasmablast expansion in MIS-C, together with elevated levels of IL-6, IL-10 and tumor necrosis factor $\alpha$, all of which can drive B cell proliferation, support the observed non-specific B cell activation in MIS-C reported here ${ }^{37-39}$. In this study, we did not observe evidence of hypergammaglobulinemia in children with MIS-C or drastic shifts in antibody glycosylation (Supplementary Fig. 12), both associated with autoimmune disease and aberrant humoral immunity. However, we found elevated responses to several respiratory common CoVs, other pathogens previously associated with $\mathrm{KD}$ or auto-antigens previously linked to aberrant MIS- $\mathrm{C}^{19}$, pointing to a potential over-activated, but not generally enhanced, humoral immune response. Interestingly, IgG1, but not IgM, titers were expanded in children with severe MIS-C, potentially indicating a predominant reactivation of previously primed B cells rather than the elicitation of novel B cell responses. Moreover, expanding IgG1 and Fc $\gamma$ R titers were highly correlated, indicating a generalized more pro-inflammatory potential within the humoral immune response, similarly to the response typically observed after a secondary recall response. Given our emerging appreciation for the presence of virus in distal tissues, including the heart and kidney, it is possible that these persisting over-active antibodies might drive tissue-localized macrophage or monocyte activation within these sites, contributing to disease activity. Given the cross-sectional nature of this study, precise insights into the mechanism of sustained inflammatory antibody production remain unclear. Emerging longitudinal studies will provide key insights into the specific events that drive B cell activation, as well as the precise antibody specificities that might contribute to tissue damage and pathology.

Taken together, our findings suggest that $\operatorname{IgA}$ and monocyteactivating antibodies play an unexpected pathological role in COVID-19 and MIS-C, respectively. Given the low probability that children are exposed to less virus that might cause milder disease in this population, the data argue that severity of disease might be linked to the reduced IgA responses in children. Conversely, monocyte-activating IgG that exhibits enhanced and persistent Fc receptor-binding capacity, rather than $\mathrm{IgA}$, in MIS-C, might contribute to organ attack via the recognition of SARS-CoV-2 or other pathogens or auto-antigens. Thus, overall, comparisons of humoral immunity in children provide clues related to the potential pathological functions of antibodies after SARS-CoV-2 infection.

\section{Online content}

Any methods, additional references, Nature Research reporting summaries, source data, extended data, supplementary information, acknowledgements, peer review information; details of author contributions and competing interests; and statements of data and code availability are available at https://doi.org/10.1038/ s41591-021-01263-3.

Received: 4 September 2020; Accepted: 25 January 2021; Published online: 12 February 2021

\section{References}

1. CDC COVID-19 Response Team. Coronavirus disease 2019 in childrenUnited States, February 12-April 2, 2020. MMWR Morb. Mortal. Wkly. Rep. 69, 422-426 (2020).

2. Bi, Q. et al. Epidemiology and transmission of COVID-19 in 391 cases and 1286 of their close contacts in Shenzhen, China: a retrospective cohort study. Lancet Infect. Dis. 20, 911-919 (2020).

3. Yonker, L. M. et al. Pediatric SARS-CoV-2: clinical presentation, infectivity, and immune responses. J. Pediatr. 227, 45-52 (2020).

4. Braun, J. et al. SARS-CoV-2-reactive T cells in healthy donors and patients with COVID-19. Nature 587, 220-224 (2020).

5. Ladner, J. T. et al. Epitope-resolved profiling of the SARS-CoV-2 antibody response identifies cross-reactivity with endemic human coronaviruses. Cell Rep. Med. 2, 100189 (2021).

6. Verdoni, L. et al. An outbreak of severe Kawasaki-like disease at the Italian epicentre of the SARS-CoV-2 epidemic: an observational cohort study. Lancet 395, 1771-1778 (2020).

7. Riphagen, S., Gomez, X., Gonzalez-Martinez, C., Wilkinson, N. \& Theocharis, P. Hyperinflammatory shock in children during COVID-19 pandemic. Lancet 395, 1607-1608 (2020).

8. Feldstein, L. R. et al. Multisystem inflammatory syndrome in U.S. children and adolescents. N. Engl. J. Med. 383, 334-346 (2020).

9. Belhadjer, Z. et al. Acute heart failure in multisystem inflammatory syndrome in children (MIS-C) in the context of global SARS-CoV-2 pandemic. Circulation 142, 429-436 (2020).

10. Zohar, T. \& Alter, G. Dissecting antibody-mediated protection against SARS-CoV-2. Nat. Rev. Immunol. 20, 392-394 (2020).

11. Atyeo, C. et al. Distinct early serological signatures track with SARS-CoV-2 survival. Immunity 53, 524-532 (2020).

12. Hamre, R., Farstad, I. N., Brandtzaeg, P. \& Morton, H. C. Expression and modulation of the human immunoglobulin A Fc receptor (CD89) and the FcR $\gamma$ chain on myeloid cells in blood and tissue. Scand. J. Immunol. 57, 506-516 (2003).

13. Veras, F. P. et al. SARS-CoV-2-triggered neutrophil extracellular traps mediate COVID-19 pathology. J. Exp. Med. 217, e20201129 (2020).

14. Laforge, $M$. et al. Tissue damage from neutrophil-induced oxidative stress in COVID-19. Nat. Rev. Immunol. 20, 515-516 (2020).

15. Witko-Sarsat, V., Rieu, P., Descamps-Latscha, B., Lesavre, P. \& Halbwachs-Mecarelli, L. Neutrophils: molecules, functions and pathophysiological aspects. Lab. Invest. 80, 617-653 (2000). 
16. Zuo, Y. et al. Neutrophil extracellular traps in COVID-19. JCI Insight 5 , e138999 (2020).

17. Weisberg, S. P. et al. Distinct antibody responses to SARS-CoV-2 in children and adults across the COVID-19 clinical spectrum. Nat. Immunol. 22, 25-31 (2020)

18. Menikou, S., Langford, P. R. \& Levin, M. Kawasaki disease: the role of immune complexes revisited. Front. Immunol. 10, 1156 (2019).

19. Consiglio, C. R. et al. The immunology of multisystem inflammatory syndrome in children with COVID-19. Cell 183, 968-981 (2020).

20. Fagarasan, S. \& Honjo, T. Intestinal IgA synthesis: regulation of front-line body defences. Nat. Rev. Immunol. 3, 63-72 (2003).

21. Woof, J. M. \& Kerr, M. A. IgA function-variations on a theme. Immunology 113, 175-177 (2004).

22. Aleyd, E. et al. IgA enhances NETosis and release of neutrophil extracellular traps by polymorphonuclear cells via Fco receptor I. J. Immunol. 192, 2374-2383 (2014)

23. Yu, H. Q. et al. Distinct features of SARS-CoV-2-specific IgA response in COVID-19 patients. Eur. Respir. J. 56, 2001526 (2020).

24. Liu, Y. et al. Neutrophil-to-lymphocyte ratio as an independent risk factor for mortality in hospitalized patients with COVID-19. J. Infect. 81, e6-e12 (2020)

25. Barnes, B. J. et al. Targeting potential drivers of COVID-19: neutrophil extracellular traps. J. Exp. Med. 217, e20200652 (2020).

26. Weemaes, C. et al. Development of immunoglobulin A in infancy and childhood. Scand. J. Immunol. 58, 642-648 (2003).

27. Pattemore, P. K. \& Jennings, L. C. in Pediatric Respiratory Medicine 2nd ed. (eds Taussig L. \& Landau, L.) 435-452 (Mosby, 2008).

28. Takahashi, K., Oharaseki, T., Yokouchi, Y., Hiruta, N. \& Naoe, S. Kawasaki disease as a systemic vasculitis in childhood. Ann. Vasc. Dis. 3, 173-181 (2010).

29. Mason, W. H., Jordan, S. C., Sakai, R., Takahashi, M. \& Bernstein, B. Circulating immune complexes in Kawasaki syndrome. Pediatr. Infect. Dis. 4, 48-51 (1985).
30. Richards, J. O. et al. Optimization of antibody binding to Fc $\gamma$ RIIa enhances macrophage phagocytosis of tumor cells. Mol. Cancer Ther. 7, 2517-2527 (2008)

31. Khor, C. C. et al. Genome-wide association study identifies FCGR2A as a susceptibility locus for Kawasaki disease. Nat. Genet. 43, 1241-1246 (2011).

32. Nagelkerke, S. Q. et al. Inhibition of Fc $\gamma$ R-mediated phagocytosis by IVIg is independent of IgG-Fc sialylation and Fc $\gamma$ RIIb in human macrophages. Blood 124, 3709-3718 (2014).

33. Abe, J. et al. Gene expression profiling of the effect of high-dose intravenous Ig in patients with Kawasaki disease. J. Immunol. 174, 5837-5845 (2005).

34. Godfred-Cato, S. et al. COVID-19-associated multisystem inflammatory syndrome in children-United States, March-July 2020. MMWR Morb. Mortal. Wkly. Rep. 69, 1074-1080 (2020).

35. Ibarrondo, F. J. et al. Rapid decay of anti-SARS-CoV-2 antibodies in persons with mild COVID-19. N. Engl. J. Med. 383, 1085-1087 (2020).

36. Long, Q. X. et al. Clinical and immunological assessment of asymptomatic SARS-CoV-2 infections. Nat. Med. 26, 1200-1204 (2020).

37. Gruber, C. N. et al. Mapping systemic inflammation and antibody responses in multisystem inflammatory syndrome in children (MIS-C). Cell 183, 982-995 (2020).

38. Diorio, C. et al. Multisystem inflammatory syndrome in children and COVID-19 are distinct presentations of SARS-CoV-2. J. Clin. Invest. 130, 5967-5975 (2020).

39. Carter, M. J. et al. Peripheral immunophenotypes in children with multisystem inflammatory syndrome associated with SARS-CoV-2 infection. Nat. Med. 26, 1701-1707 (2020).

Publisher's note Springer Nature remains neutral with regard to jurisdictional claims in published maps and institutional affiliations.

(C) The Author(s), under exclusive licence to Springer Nature America, Inc. 2021 


\section{Methods}

Cohort. Plasma samples were obtained from pediatric and adult patients at Massachusetts General Hospital (MGH) under the institutional review board (IRB)-approved 'MGH Pediatric COVID-19 Biorepository' (no. 2020P000955). Additionally, plasma samples from adult patients were collected under the IRB-approved 'MGH COVID-19 Biorepository' (no. 2020P000804) and the 'Biorepository for samples from those at increased risk for or infected with SARS-CoV-2' (no. 2020P000849). Informed consent, and assent when appropriate, were obtained in accordance with IRB guidelines from the patients or parents/ guardians before study enrollment. Informed consent of the pediatric cohort included approval to publish results from research studies using de-identified patient samples. A detailed description of the pediatric cohort characteristics was previously published ${ }^{3,40}$. Patients were diagnosed with COVID-19-related symptoms and had positive PCR and/or serology for SARS-CoV-2. MIS-C was diagnosed according to CDC criteria. Severe MIS-C was defined by the presence of hypotension or cardiac abnormalities that required intervention, including steroids, IVIG and/or anakinra (Supplementary Table 2). All analyzed samples were taken before treatment with IVIG. If not stated otherwise, only one time point per individual was available, and blood was drawn during visit in the outpatient clinic for mild cases (adult cohort was collected 0-52 d after symptom onset; median, $7 \mathrm{~d}$; pediatric cohort was collected 0-21 d after symptom onset; median, $3 \mathrm{~d}$ ) and convalescent cases (collected 11-38 d after symptom onset; median, $27 \mathrm{~d}$ ) or as regular blood collection during hospital stay for patients with severe COVID-19 (collected 10-37 d after symptom onset; median, 16 d) and patients with MIS-C (collected 2-23 d after MIS-C symptom onset; median, $3 \mathrm{~d}$ ) (Supplementary Table 1). This study was approved by the MGH Human Subjects Institutional Review Board.

Antigens and biotinylation. The RBDs of SARS2-CoV-2 and CoV strains NL63, HKU1, OC43 and 229 were generously provided by Aaron Schmidt. SARS2-CoV2-S was provided by LakePharma. SARS2-CoV2-NC (Aalto Bio Reagents), flu hemagglutinins and EBV p18 (both from ImmuneTech), RSV postF (generously provided by Barney Graham), measles (Bio-Rad), ERV-W antigen and translocator protein (TSPO) (both from Abnova), pertussix toxin (List Biological Laboratories), CD105 (Novus Biologicals) and RBPJK (OriGene) were all purchased from the different vendors.

If indicated, antigens were biotinylated using Sulfo-NHS LCLC biotin (Thermo Fisher Scientific), and excessive biotin was removed with Zeba Spin Desalting Columns (7-kDa cutoff, Thermo Fisher Scientific).

IgG subclass, isotype and $\mathrm{Fc} \gamma \mathrm{R}$ binding. Antigen-specific antibody subclass and isotypes and $\mathrm{F} c \gamma \mathrm{R}$ binding were further analyzed by Luminex multiplexing. The antigens were coupled to magnetic Luminex beads by carbodiimide-NHS ester coupling with an individual region per antigen. Coupled beads were incubated with different plasma dilutions (between 1:100 and 1:1,000 depending on the secondary reagent) for $2 \mathrm{~h}$ at room temperature in 384-well plates (Greiner Bio-One). Unbound antibodies were washed away, and subclasses and isotypes were detected with a respective phycoerythrin (PE)-conjugated antibody at a 1:100 dilution (SouthernBiotech; Life Sciences Reporting Summary). For the Fc $\gamma$ R binding, a respective PE-streptavidin (Agilent Technologies) coupled recombinant and biotinylated human $\mathrm{Fc} \gamma \mathrm{R}$ protein was used as a secondary probe. After 1-h incubation, excessive secondary reagent was washed away, and the relative antibody concentration per antigen was determined on an iQue analyzer (Intellicyt). Samples were defined seropositive when they had detectable titer (by Luminex) for IgG1, IgM and/or IgA1, and a negative cutoff was defined as the average value of SARS-CoV-2-negative samples plus five times the s.d. Luminex results for IgG1 were validated by RBD-IgG enzyme-linked immunosorbent assay (ELISA) as previously described (Supplementary Fig. 13) ${ }^{41}$.

Antibody-dependent complement deposition. Antibody-dependent complement deposition (ADCD) was assessed as described previously ${ }^{42}$. In brief, biotinylated antigen was coupled to fluorescence NeutrAvidin beads (Thermo Fisher Scientific). Plasma antibodies were diluted 1:10 in $0.1 \%$ BSA and incubated with the coupled antigen beads for $2 \mathrm{~h}$ at $37^{\circ} \mathrm{C}$. Beads were washed and incubated with complement factors from guinea pig for $20 \mathrm{~min}$ at $37^{\circ} \mathrm{C}$. The complement reaction was then stopped by washing with $15 \mathrm{mM}$ EDTA in PBS. C3 deposition on the beads was detected with a 1:100 diluted FITC-conjugated anti-guinea pig C3 polyclonal antibody (MP Biomedicals), and relative $\mathrm{C} 3$ deposition was analyzed by flow cytometry.

\section{Antibody-dependent neutrophil phagocytosis. For antibody-dependent} neutrophil phagocytosis (ADNP), HL-60 cells were differentiated into neutrophils in media containing $1.2 \%$ DMSO for $7 \mathrm{~d}$. On the day of the assay, biotinylated antigens were incubated with NeutrAvidin beads and ICs formed by incubation with 1:100 diluted plasma for $2 \mathrm{~h}$ at $37^{\circ} \mathrm{C}$ in 96 -well plates (Greiner Bio-One). Differentiated HL-60 cells were added afterwards and incubated for $20 \mathrm{~h}$ at $37^{\circ} \mathrm{C}$. Neutrophils were surface stained against CD11b (1:100, BD Biosciences, clone: ICRF44), fixed with $4 \%$ paraformaldehyde and analyzed on an iQue flow cytometer. Phagocytosis score was calculated as the product of frequency bead-positive CD11b neutrophils and bead fluorescent intensity using ForeCyt Standard Edition 8.1 software (Supplementary Fig. 14).

Antibody-dependent cellular phagocytosis. THP-1 monocyte phagocytosis was performed as previously described ${ }^{43}$. Briefly, biotinylated antigens were conjugated to NeutrAvidin beads and incubated with 1:100 diluted plasma samples. THP-1 monocytes $(0.25 \mathrm{M}$ cells per well) were added to the ICs and incubated for $16 \mathrm{~h}$ at $37^{\circ} \mathrm{C}$, fixed with $4 \%$ paraformaldehyde and analyzed by flow cytometry (Supplementary Fig. 14)

Plasma IgA and IgG depletion. IgA was depleted from human plasma samples using CaptureSelect IgA Affinity Matrix, and IgG was depleted with Protein A/G Agarose (Thermo Fisher Scientific). The capture matrices were washed three times with PBS and incubated overnight with 1:5 diluted plasma samples in a low-protein-binding MultiScreen filter plate (Millipore). Depleted plasma was recovered by centrifugation of the filter plate, and depletion was confirmed by ELISA. Non-depleted plasma was treated similarly but without Affinity Matrix.

Primary neutrophil ADNP. Neutrophil phagocytic activity of IgA-depleted plasma samples was tested on primary human neutrophils ${ }^{44}$. Biotinylated antigens were coupled to fluorescent NeutrAvidin beads and incubated 1:100 with diluted plasma. Primary neutrophils were incubated with ICs for $1 \mathrm{~h}$ at $37^{\circ} \mathrm{C}$. Next, cells were stained for surface CD66b expression with a 1:100 diluted antibody (BioLegend, clone: G10F5), fixed with $4 \%$ paraformaldehyde and analyzed by flow cytometry, and phagocytosis score was calculated as described above (Supplementary Fig. 14).

Secondary neutrophil assays. Blood neutrophils of healthy donors were isolated using EasySep Direct Human Neutrophil Isolation Kit (STEMCELL Technologies). Neutrophils were stimulated with bead-based ICs, and supernatants were harvested after $4 \mathrm{~h}$. Supernatants were diluted 1:5, and release of MPO (Thermo Fisher Scientific), MMP-9 and lactoferrin (Abcam) were measured using the human ELISA kits according to manufacturer instructions. Cytokines were detected in undiluted supernatant using a custom-made multiplex cytokine kit (Thermo Fisher Scientific).

Plasma isotype ELISA. Plasma IgG, IgM and IgA concentrations were analyzed by ELISA. Respective goat anti-human capture antibody against IgG, IgM or IgA $\left(5 \mu \mathrm{g} \mathrm{ml}^{-1}\right)$ (all Bethyl Laboratories; Life Sciences Reporting Summary) were coated to a MaxiSorp 384-well ELISA plate (Thermo Fisher Scientific). Unspecific binding sites were blocked with 5\% BSA. Plasma samples were diluted 1:500,000 for IgG and 1:50,000 for IgM and IgA. After sample incubation, IgG, IgM or IgA was probed with 1:10,000 of horseradish peroxidase-conjugated goat anti-human IgG, IgM or IgA antibody (all Bethyl Laboratories). The ELISA was developed with $3,3^{\prime}, 5,5^{\prime}$-tetramethylbenzidine (Thermo Fisher Scientific), and the reaction was stopped with sulfuric acid. The absorbance was acquired at $450 / 570 \mathrm{~nm}$ on a Tecan Infinite M1000 Pro plate reader and Tecan i-control v3.4.2 software (both BioTek). Concentrations were calculated from a two-fold serial dilution curve pooled normal IgG (Bethyl Laboratories; Life Sciences Reporting Summary) or IgM (Sigma-Aldrich) starting at $125 \mathrm{ng} \mathrm{ml}^{-1}$.

IgG Fc glycosylation. Plasma samples were diluted 1:10 with PBS and incubated overnight at $4^{\circ} \mathrm{C}$ with magnetic protein $\mathrm{G}$ beads (Millipore). Fab fragments were enzymatically separated from the protein G-bound Fc parts using IdeZ (NEB). IgG Fc glycans were released from the protein and APTS labeled with GlycanAssure APTS Kit (Thermo Fisher Scientific) and analyzed on a 3500xL Genetic Analyzer (Thermo Fisher Scientific) capillary electrophoresis instrument. Glycans were assigned based on retention times of known standard glycans with Glycan Acquisition Software Version 3500 v1.0.3 and Glycan Analysis Software v1.1 as described previously ${ }^{45}$.

Computational analysis. Microsoft Excel 365 was used to compile experimental data and patient information. For each paired group, we collected all the measurements from Luminex and functional profiling antibody assays; we eliminated features with missing values across samples. As a quality filter, we required features to have values across samples greater than $65 \%$ compared to PBS control. The filtered data from Luminex were $\log$ transformed by $\log _{10}$ function, and all the selected features were scaled and centered.

To visualize relationships between measurements and labels qualitatively, we employed UMAP ${ }^{46}$-based methods to compress this high-dimensional serological data into a two-dimensional space. First, principal components that explained more than $90 \%$ of the variance were extracted by principal component analysis ${ }^{47}$ using the 'prcomp' function in R package 'stats' (v4.0.3). Next, the selected principal components were mapped into a two-dimensional space through the UMAP technique implemented using the R package 'umap' (v0.2.7.0) with fine-tuning parameters (neighbor $=10$, min.dist $=0.1$ ).

Classification models were trained to distinguish different paired groups with a minimal set of features. First, we applied the LASSO feature selection algorithm to extract significant features ${ }^{48}$. We ran LASSO ten times on the whole dataset and identified the features chosen in more than $80 \%$ of the repetitions, which were implemented in the function 'select_lasso' in systemseRology R package (v1.0) 
(https://github.com/LoosC/systemsseRology). A PLS-DA classifier was then trained using the extracted features. Model performance was evaluated by five-fold cross-validation, and negative control models were constructed from permuted data with multiple iterations. The permuted control models were generated 20 times by shuffling labels randomly for each repetition. Predicted and true outcomes were compared to determine cross-validation accuracy. The exact $P$ values were calculated as the tail probability of the true value within the control distributions. For PLS-DA, we used the 'opls' function in ropls R package (v1.22.0) for classification and functions in the systemsseRology R package for the purpose of visualization.

We used correlation networks to visualize the additional humoral immune features significantly associated with the selected minimal features, offering enhanced insights into biological mechanisms. Antibody features that were significantly $(P<0.05)$ correlated with a Benjamini-Hochberg correction to the final selected PLS-DA features were defined as co-correlates. Significant Spearman correlations about a threshold of $|\mathrm{r}|>0.7$ were visualized within the networks.

For implementation, Spearman correlation coefficients were calculated using the 'rcorr' function in the 'Hmisc' package (v4.4.2), and the $P$ values were corrected by Benjamini-Hochberg correction in the 'stats' package (v4.0.3). Finally, the correlation networks were properly laid out and visualized using the 'ggraph' (v2.0.4) and 'igraph' (v1.2.6) packages with later manual adjustment using Adobe Illustrator (v2020)

Statistical analysis. If not stated otherwise, we assumed non-normal distributions, and violin plots were generated and statistical differences between two groups were calculated using a two-sided Mann-Whitney test. Solid lines in the violin plot indicate 25 th or 75 th quartile, and the dashed line indicates the median value. To compare multiple groups, a Kruskal-Wallis test was used with a Dunnett test correcting for multiple comparisons in GraphPad Prism v8 (significance levels: $\left.{ }^{\star} P<0.05,{ }^{* *} P<0.01,{ }^{* *} P<0.001,{ }^{* * *} P<=0.0001\right)$. Flower plots were visualized with the ggplot package (v0.7); correlation chord diagrams were plotted using the circlize package (v0.4.1) in R (v4.0.1) and R Studio (v1.3); and the average of the $Z$-scored data per variable and group is shown.

Life Sciences Reporting Summary. Additional information about this study is available in the Life Sciences Reporting Summary, which is published online along with this paper.

\section{Data availability}

All relevant data are included in this manuscript. Source data are provided with this paper.

\section{Code availability}

There was no specific custom code used in this manuscript. All code is publicly available, and the source is indicated in the text and/or the Methods section. Scripts will be made available upon reasonable request. Source data are provided with this paper.

\section{References}

40. Lima, R. et al. Establishment of a pediatric COVID-19 biorepository: unique considerations and opportunities for studying the impact of the COVID-19 pandemic on children. BMC Med. Res. Methodol. 20, 228 (2020).

41. Roy, V. et al. SARS-CoV-2-specific ELISA development. J. Immunol. Methods 484-485, 112832 (2020).

42. Fischinger, S. et al. A high-throughput, bead-based, antigen-specific assay to assess the ability of antibodies to induce complement activation. J. Immunol. Methods 473, 112630 (2019).
43. Ackerman, M. E. et al. A robust, high-throughput assay to determine the phagocytic activity of clinical antibody samples. J. Immunol. Methods 366, 8-19 (2011).

44. Karsten, C. B. et al. A versatile high-throughput assay to characterize antibody-mediated neutrophil phagocytosis. J. Immunol. Methods 471, 46-56 (2019).

45. Mahan, A. E. et al. A method for high-throughput, sensitive analysis of IgG Fc and Fab glycosylation by capillary electrophoresis. J. Immunol. Methods 417, 34-44 (2015).

46. McInnes, L., Healy, J. \& Melville, J. UMAP: uniform manifold approximation and projection for dimension reduction. Preprint at https://arxiv.org/ abs/1802.03426 (2018).

47. Wold, S., Esbensen, K. \& Geladi, P. Principal component analysis. Chemom. Intell. Lab. Syst. 2, 37-52 (1987).

48. Tibshirani, R. Regression shrinkage and selection via the lasso: a retrospective. J. R. Stat. Soc. B 73, 273-282 (2011).

\section{Acknowledgements}

L.M.Y. received funding from the National Heart, Lung and Blood Institute (5K08HL143183) and the Cystic Fibrosis Foundation (YONKER18Q0). D.A.L. was partially supported by the National Institute for Allergy and Infectious Diseases (U19 AI135995). We thank N. Zimmerman, M. and L. Schwartz, an anonymous donor (financial support), T. and S. Ragon and the SAMANA Kay MGH Research Scholars Award for their support. We would also like to thank Y. Cai and B. Chen for S protein production efforts and J. Feldman, B. M. Hauser, T. Caradonna and A. Schmidt for generating receptor-binding domain antigens. We acknowledge support from the Ragon Institute of MGH, MIT, the Massachusetts Consortium on Pathogen Readiness, the NIH (3R37AI080289-11S1, R01AI146785, U19AI42790-01, U19AI135995-02, U19AI42790-01, CIVIC75N93019C00052), Centers for Disease Control and Prevention U01CK000490, NIH SeroNet U01CA260476, the Musk Foundation, and the Gates Foundation Global Health Vaccine Accelerator Platform funding (OPP1146996). We would also like to thank X. Yu, A. Piechocka-Trocha and K. Lefteri for their support on the collection and processing of adult MassCPR cohorts.

\section{Author contributions}

Y.C.B., L.M.Y. and G.A. analyzed and interpreted the data. Y.C.B., T.Z., S.F., C.A., J.B., J.K., A.R.H. and D.I. performed experiments. C.W. and D.A.L. performed the computational analysis. A.G.E., A.F., L.R.B., E.J.N., A.E.W., E.W.B., E.T.R., R.C., B.D.J. and L.M.Y. collected the samples and supervised and managed the clinical data. E.S.F. produced SARS-CoV-2 S antigen. L.M.Y. and G.A. supervised the project. Y.C.B. and G.A. drafted the manuscript. All authors critically reviewed the manuscript.

\section{Competing interests}

G.A. is a founder of Seromyx Systems, a company developing a platform technology that describes the antibody immune response. G.A.'s interests were reviewed and are managed by Massachusetts General Hospital and Partners HealthCare in accordance with their conflict of interest policies. All other authors have declared that no conflicts of interest exist.

\section{Additional information}

Supplementary information The online version contains supplementary material available at https://doi.org/10.1038/s41591-021-01263-3.

Correspondence and requests for materials should be addressed to L.M.Y. or G.A.

Reprints and permissions information is available at www.nature.com/reprints. 


\section{Reporting Summary}

Nature Research wishes to improve the reproducibility of the work that we publish. This form provides structure for consistency and transparency in reporting. For further information on Nature Research policies, see our Editorial Policies and the Editorial Policy Checklist.

\section{Statistics}

For all statistical analyses, confirm that the following items are present in the figure legend, table legend, main text, or Methods section.

$\mathrm{n} / \mathrm{a}$ Confirmed

$\bigotimes$ The exact sample size $(n)$ for each experimental group/condition, given as a discrete number and unit of measurement

\ A statement on whether measurements were taken from distinct samples or whether the same sample was measured repeatedly

The statistical test(s) used AND whether they are one- or two-sided

Only common tests should be described solely by name; describe more complex techniques in the Methods section.

\A description of all covariates tested

$\square$ A description of any assumptions or corrections, such as tests of normality and adjustment for multiple comparisons

A full description of the statistical parameters including central tendency (e.g. means) or other basic estimates (e.g. regression coefficient)

AND variation (e.g. standard deviation) or associated estimates of uncertainty (e.g. confidence intervals)

$\square$ For null hypothesis testing, the test statistic (e.g. $F, t, r$ ) with confidence intervals, effect sizes, degrees of freedom and $P$ value noted

Give $P$ values as exact values whenever suitable.

Х $\square$ For Bayesian analysis, information on the choice of priors and Markov chain Monte Carlo settings

$\triangle \square$ For hierarchical and complex designs, identification of the appropriate level for tests and full reporting of outcomes

$\triangle(\square$ Estimates of effect sizes (e.g. Cohen's $d$, Pearson's $r$ ), indicating how they were calculated

\section{Our web collection on statistics for biologists contains articles on many of the points above.}

\section{Software and code}

Policy information about availability of computer code

Data collection ForeCyt ${ }^{\circledR}$ Standard Edition 8.1 was used to collect Luminex, ADNP, ADCP and ADCD assay.

Glycan Acquisition Software Version 3500 V1.0.3 was used to collect glycan traces

Tecan-i-control V.3.4.2 was used to collect ELISA data.

Data analysis Microsoft Excel 365 was used to compile experimental data and patient information.

Violin plots and statistical analysis was performed with GraphPad Prism v.8.4.2.

Flower plots were generated in RStudio (v.1.3 and R v.4.0) using 'ggplot2' package (v.3.3).

GlycanAssure Data Analysis software v.1.1 was used to analyze glycan traces.

PCA in 'stats' package (4.0.3) was used to reduce the multivariate features into independent components and the number of components representing more than $90 \%$ of variances were then used for Umap visualization.

Umap implemented in 'umap' package (0.2.7.0) was used to map the multivariate features into two dimension with the parameter (neighbor

$=10$, min.dist $=0.1$ ) and then the mapped data were visualized using 'ggplot2' package (v.3.3).

The integrated functions in 'systemRology' package(https://github.com/LoosC/systemsseRology) (1.0) were used to perform feature selection and partial least-squares discriminant analysis(PLS-DA) implemented in 'ropls' package (1.22.0).

Spearman correlation coefficients were calculated using ' $r$ corr' function in 'Hmisc' package (4.4.2) and the p values were corrected by 'Benjamini-Hochberg' correction implemented in 'stats' package (4.0.3). The correlation networks were properly lay-outed and visualized using 'ggraph' (2.0.4) and 'igraph' (1.2.6) package with later manual adjustment using Adobe Illustrator (2020).

For manuscripts utilizing custom algorithms or software that are central to the research but not yet described in published literature, software must be made available to editors and

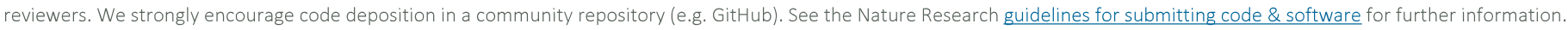


Policy information about availability of data

All manuscripts must include a data availability statement. This statement should provide the following information, where applicable:

- Accession codes, unique identifiers, or web links for publicly available datasets

- A list of figures that have associated raw data

- A description of any restrictions on data availability

All relevant data are included in this manuscript. Source data are provided with this paper.

\section{Field-specific reporting}

Please select the one below that is the best fit for your research. If you are not sure, read the appropriate sections before making your selection.

Х Life sciences

Behavioural \& social sciences

Ecological, evolutionary \& environmental sciences

For a reference copy of the document with all sections, see nature.com/documents/nr-reporting-summary-flat.pdf

\section{Life sciences study design}

All studies must disclose on these points even when the disclosure is negative.

Sample size No sample size calculation was performed. We included all pediatric COVID-19 or MIS-C patients treated at Massachusetts General Hospital between March and June 2020 who gave informed consent. Adult patients were selected based on the pediatric sample size. Based on experiences from previous studies, sample sizes are assumed to be sufficient.

Data exclusions SARS-CoV-2 seronegative samples were excluded from the multivariate analyses and univariate analyses of ADCD, ADCP and ADNP. Samples were defined sero-positive when they had detectable titer (by Luminex) for IgG1, IgM and/or IgA1 and a negative cut-off was defined as the average value negative plasma samples plus 5 times the standard deviation.

For multivariate analysis, the feature, whose proportion across samples was less than $65 \%$ compared with the value of PBS control, was excluded. This exclusion-criteria was pre-established.

Replication All experiments were run in duplicates and Luminex and functional assays (ADCD, ADNP and ADCD) repeated for most of the samples. Results between repeats were comparable.

Randomization Samples were randomly distributed in 96 well plates

Blinding

Investigators were blinded during data collection. Group allocation had to be revealed to perform the data analysis.

\section{Reporting for specific materials, systems and methods}

We require information from authors about some types of materials, experimental systems and methods used in many studies. Here, indicate whether each material, system or method listed is relevant to your study. If you are not sure if a list item applies to your research, read the appropriate section before selecting a response.

\begin{tabular}{|c|c|c|c|}
\hline \multicolumn{2}{|r|}{ Materials \& experimental systems } & \multicolumn{2}{|c|}{ Methods } \\
\hline $\mathrm{n} / \mathrm{a}$ & Involved in the study & $\mathrm{n} / \mathrm{a}$ & Involved in the study \\
\hline & $\bigotimes$ Antibodies & $\bigotimes$ & $\square$ ChIP-seq \\
\hline 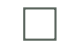 & Eukaryotic cell lines & $\square$ & Flow cytometry \\
\hline Х & $\square$ Palaeontology and archaeology & Х & $\square$ MRI-based neuroimaging \\
\hline Х & Animals and other organisms & & \\
\hline & \ Human research participants & & \\
\hline Х & $\square$ Clinical data & & \\
\hline$\bigotimes$ & $\square$ Dual use research of concern & & \\
\hline
\end{tabular}

\section{Antibodies}

Antibodies used

1.Mouse Anti-Human IgG1-PE (Southern-Biotech, \#9054-09, clone:HP6001)

2. Mouse Anti-Human IgG2-PE (Southern-Biotech, \#9060-09, clone:31-7-4)

3. Mouse Anti-Human IgG3-PE (Southern-Biotech, \#9210-09, clone:HP6050)

4. Mouse Anti-Human IgG4-PE (Southern-Biotech, \#9200-09, clone:HP6025

5. Mouse Anti-Human IgM-PE (Southern-Biotech, \#9020-09, clone:SA-DA4)

6. Mouse Anti-Human IgA1-PE (Southern-Biotech, \#9130-09, clone: B3506B4)

7. Mouse Anti-Human IgA2-PE (Southern-Biotech, \#9140-09, clone: A9604D2) 
8. anti-human CD11b BV605 (BD, \#562721, Clone ICRF44)

9. Anti-guinea pig complement C3 goat IgG fraction (MP Biomedical, \#855385. polyclonal)

10. anti-human CD66b Pacific Blue (Biolegend, \#305112, clone G10F5)

11. anti-human IgG-Fc-HRP (Bethyl Labs, \#A80-148P, polyclonal)

12. anti-human IgM-Fc-HRP (Bethyl Labs, \#A80-100P, polyclonal)

13. anti-human IgA-Fc-HRP (Bethyl Labs, \#A80-102P, polyclonal)

14. anti-human IgG-Fc (Bethyl Labs, \#A80-148A, polyclonal)

15. anti-human IgM-Fc (Bethyl Labs, \#A80-100, polyclonal)

16. anti-human IgA-Fc (Bethyl Labs, \#A80-102, polyclonal)

Validation

All antibodies are well established and quality controlled by the manufacturer. Additional information and references can be obtained on the company websites.

The use of antibodies 1-4 was previously validated: Brown EP, Licht AF, Dugast AS, Choi I, Bailey-Kellogg C, Alter G, et al. Highthroughput, multiplexed IgG subclassing of antigen-specific antibodies from clinical samples. J Immunol Methods.

2012;386(1-2):117-23.

Antibody 9 was described here: Fischinger, S., J. K. Fallon, A. R. Michell, T. Broge, T. J. Suscovich, H. Streeck, and G. Alter. 2019. 'A

high-throughput, bead-based, antigen-specific assay to assess the ability of antibodies to induce complement activation', J Immunol Methods, 473: 112630.

Antibody 10: Karsten, C. B., N. Mehta, S. A. Shin, T. J. Diefenbach, M. D. Slein, W. Karpinski, E. B. Irvine, T. Broge, T. J. Suscovich, and G. Alter. 2019. 'A versatile high-throughput assay to characterize antibody-mediated neutrophil phagocytosis', J Immunol Methods, 471: 46-56.

\section{Eukaryotic cell lines}

Policy information about cell lines

Cell line source(s)

HL60 cells ( promyeloblast)

THP-1 cells (human acute monocytic leukemia)

Authentication

None of the cell lines used were authenticated.

Mycoplasma contamination

Cell lines were not tested for mycoplasma contamination

Commonly misidentified lines

(See ICLAC register)

No commonly misidentified lines were used.

\section{Human research participants}

\section{Policy information about studies involving human research participants}

Population characteristics

Recruitment

Ethics oversight
We analyzed 25 children/young adults (ages 0-20 years, median $=15 y$ ) and 34 adults ages $22-76$ years, median $=33.5 y, 52 \%$ female) diagnosed with mild disease, and 26 hospitalized adults (age between 30-79, 53\% female) with severe SARS-CoV-2 infection. Additionally, 17 children/young-adults ( 0 -22 years, median $3.5 y, 17 \%$ female) diagnosed with MIS-C and 18 SARSCoV-2 convalescent adults (ages: $27-58 y$, median: $33 y, 55 \%$ female)

Patients visited the respiratory illness clinic (RIC) or were hospitalized at a MassGeneralBrigham site in Massachusetts, USA. There was no bias in the patient recruitment.

The study was approved by the MassGeneralBrigham IRB. Study number \#2020P000955, \#2020P000804 and \#2020P000849.

Note that full information on the approval of the study protocol must also be provided in the manuscript.

\section{Flow Cytometry}

Plots

Confirm that:

\The axis labels state the marker and fluorochrome used (e.g. CD4-FITC).

\The axis scales are clearly visible. Include numbers along axes only for bottom left plot of group (a 'group' is an analysis of identical markers).

\All plots are contour plots with outliers or pseudocolor plots.

$\bigotimes$ A numerical value for number of cells or percentage (with statistics) is provided.

\section{Methodology}

Sample preparation
ADNP

After phagocytosis incubation HL-60 cells were pelleted and surface stained with anti-CD11b OR primary neutrophils were stained with anti-CD66b. Cells were washed with PBS and fixed with $4 \%$ para-formaldehyde. Cells were washed with PBS after fixation, resuspended in PBS and stored at $4 \mathrm{C}$ protected from light for up to $24 \mathrm{~h}$, if flow cytometric analysis was not immediately possible. 
THP-1 cells were pelleted and fixed with $4 \%$ para-formaldehyde and washed with PBS after fixation. Cells were then resuspended in PBS and stored at $4 \mathrm{C}$ protected from light for up to $24 \mathrm{~h}$, if immediate flow cytometric analysis was not immediately possible.

Instrument

Software

Cell population abundance

Gating strategy
IntelliCyt ${ }^{\circledR}$ iQue Screener PLUS

ForeCyt ${ }^{\circledR}$ Standard Edition 8.1 was used to collect and analyze the data

Differentiated HL-60 neutrophils were identified by CD11b surface expression. Usually $>90 \%$ of single cells were CD11b positive.

Primary neutrophils were identified by CD66b surface expression. CD66b expression was donor dependent bu usually $>95 \%$ within the single cell gate.

All single cells were considered THP-1 cells.

All events were gated for granulocutes using FSC-H and SSC-H and single cells subsequently selected using SSC-A and SSC-H. For ADNP assay: HL-60 differentiated neutrophils were selected by CD11b positivity or primary neutrophils selected for CD66b expression. Phagocytic cells identified in the BL4-H channel.

Phagocytic THP-1 cells were directly determined in the single cell population.

$\bigotimes$ Tick this box to confirm that a figure exemplifying the gating strategy is provided in the Supplementary Information. 